\section{Case Reports in Dermatology}

Case Rep Dermatol 2017;9:19-25

DOI: $10.1159 / 000473872$

Publisned onime: TVlay Z2, 2017 (c) 2017 The Author(s)

Published by S. Karger AG, Basel www.karger.com/cde

This article is licensed under the Creative Commons Attribution-NonCommercial 4.0 International License (CC BY-NC) (http://www.karger.com/Services/OpenAccessLicense). Usage and distribution for commercial purposes requires written permission.

\title{
Successful Treatment of Chronic Staphylococcus aureus-Related Dermatoses with the Topical Endolysin Staphefekt SA.100: A Report of 3 Cases
}

\author{
Joan E.E. Totté Martijn B. van Doorn Suzanne G.M.A. Pasmans \\ Department of Dermatology, Erasmus MC University Medical Center Rotterdam, \\ Rotterdam, The Netherlands
}

\section{Keywords}

Staphefekt · Staphylococcus aureus · Endolysin · Folliculitis · Atopic dermatitis · Antibiotic resistance
Abstract
Staphylococcus aureus plays an important role in skin and soft tissue infections and contrib- utes to the pathophysiology of complex skin disorders such as atopic dermatitis. Bacterial resistance against commonly used antibiotics has increased considerably in the last decades demanding alternative treatment approaches. We present 3 cases where patients with chron- ic and recurrent $S$. aureus-related dermatoses were successfully treated with Staphefekt SA.100. Staphefekt SA.100 is a recombinant phage endolysin for topical skin application that specifically targets both methicillin-sensitive and methicillin-resistant $S$. aureus. As a conse-

\footnotetext{
KARGER

Joan E.E. Totté

Department of Dermatology, Erasmus MC University Medical Center Rotterdam

Burgemeester s' Jacobplein 51

NL-3015 CA Rotterdam (The Netherlands)

E-Mail j.totte@erasmusmc.nl

Suzanne G.M.A. Pasmans

Department of Pediatric Dermatology, Sophia Children's Hospital

Erasmus MC University Medical Center Rotterdam

Wytemaweg 80, NL-3015 CN Rotterdam (The Netherlands)

E-Mail s.pasmans@erasmusmc.nl
} 
quence of its specific mechanism of action, bacterial resistance is unlikely to develop. In our 3 cases, resistance induction was not observed. Our results indicate that targeted treatment with Staphefekt might be an attractive alternative for (long-term) classical antibiotic therapy, and confirmatory randomized controlled trials are warranted to evaluate its clinical efficacy and safety.

\section{Introduction}

Staphylococcus aureus is the most common microbe causing skin and soft tissue infections and contributes to the pathophysiology of complex skin disorders such as atopic dermatitis [1-3]. As antibiotic resistance against classical antibiotics is rapidly emerging, alternative treatment options are desperately needed [4-6]. Staphefekt SA.100 is a recombinant phage endolysin for topical skin application that specifically binds to the cell wall of S. aureus and cleaves bonds in the peptidoglycan wall, resulting in bacterial cell death [7]. In vitro studies showed that Staphefekt exclusively targets $S$. aureus without affecting other (commensal) skin bacteria. Induction of resistance was not observed and is also not expected due to its specific mechanism of action [7-9]. Staphefekt is currently registered as a (class 1) medical device in Europe and is available in a cetomacrogol-based cream and in a gel as over-the-counter treatment. Evidence for its clinical efficacy is currently based on questionnaire studies and patient reviews only (Table 1) [8-10]. In this report we describe 3 cases with difficult-to-treat chronic or recurrent $S$. aureus-related dermatoses requiring antibiotic therapy that were successfully treated with Staphefekt SA.100 in our clinic.

\section{Case Presentation}

\section{Case 1}

The first patient is a 23-year-old male with a history of osteosarcoma at the age of 16 and acne vulgaris of the face and trunk. He presented with an extensive papulopustular eruption on the trunk consistent with bacterial folliculitis. Consecutive empirical treatments with clarithromycin $500 \mathrm{mg}$ once daily combined with Betadine scrub resulted in moderate improvement. However, after discontinuing treatment the symptoms recurred within 3 days. Taking into consideration the patient's reluctance to use continuous oral antibiotics and after discussing the alternative (on- and off-label) treatment options, our patient decided to try a short course of Staphefekt. He applied the cream twice daily on the affected skin for 2 weeks. At his return visit he reported a strong decrease of inflammatory symptoms that started within a few days. This triggered us to further investigate the clinical effect of Staphefekt using clinical photographs and bacterial cultures in this and 2 other cases. One week after he had stopped treatment with Staphefekt, the papules and pustules recurred and were similar to those at the baseline (visit 1; Fig. 1a). Staphefekt was restarted twice daily, again resulting in a clear decrease in the number of the papules and pustules observed 4 weeks later (visit 2). This improvement continued up to visit 3 after another 4 weeks (Fig. 1b). Nasal cultures showed persistent carriage of $S$. aureus. Skin cultures were only taken at visits 2 
and 3 with a positive culture at visit 3 . An extra skin culture was taken 2 months later (visit 4) to determine minimal inhibitory concentrations of Staphefekt. The minimal inhibitory concentrations of the cultured $S$. aureus strain at visits 3 and 4 was stable, indicating the absence of resistance during long-term daily therapy.

\section{Case 2}

The second patient, a 63-year-old male, was diagnosed with a secondary bacterial infection (impetigo) of his nummular eczema, mainly located at the face and the extremities. The diagnosis was confirmed by histopathology and S. aureus cultured from a lesion. Treatment with clarithromycin $250 \mathrm{mg}$ twice daily during 7 days, combined with topical fusidic acid cream, did not result in clinical improvement. Oral prednisolone $30 \mathrm{mg}$ once daily for 2 weeks combined with flucloxacillin $500 \mathrm{mg} 4$ times daily during 7 days resulted in clinical improvement. However, 2 days after the treatment was stopped, the symptoms recurred (Fig. 2a). After discussing the alternative treatment options, Staphefekt cream was started twice daily on the affected skin of the face and extremities, and the extremities were additionally treated with betamethasone ointment once daily. The patient reported improvement of the superinfection within 1 week. His symptoms did not recur over a treatment period of more than 12 weeks (Fig. $2 \mathrm{~b}$ ), and he did not report any adverse effects. Although the superinfection had cleared, the underlying eczema at the extremities remained. His eczema gradually improved after the treatment with betamethasone ointment was replaced with tacrolimus $0.1 \%$ ointment in tar $10 \%$ (solutio carbonis detergens) 1 month later. Cultures from the facial skin lesions and nose collected at baseline, and after 6 and 12 weeks of treatment were all positive for $S$. aureus.

\section{Case 3}

The third case is a 23-year-old man who presented with a bacterial folliculitis of the lower legs. S. aureus was cultured from a pustule but also from his nose and perineum. The patient was diagnosed with nummular eczema mainly at the extremities, which was active at the time of presentation. His medical history comprised biliary atresia followed by a liver transplant and chronic use of oral immunosuppressive medication. Class 2-3 topical corticosteroids, topical tacrolimus, and an emollient were prescribed to treat the eczema. The folliculitis was treated with topical fucidic acid cream, oral flucloxacillin for 1 week, and Betadine scrub, resulting in an improvement of the symptoms. An exacerbation 6 months later with follicular pustules at the lower legs was treated again with flucloxacillin but 2 months later the folliculitis recurred again, including extended perifollicular pustules that evolved to large confluent plaques at the lower legs (visit 1). After discussing the alternative treatment options, we started topical Staphefekt twice daily. Three weeks later (visit 2) he reported a strong improvement of his symptoms starting 4 days after initiation of the treatment. Clinical examination at this time showed the remaining extensive eczematous lesions, but pustules were clearly absent and the redness of the lesions was significantly reduced. With continued treatment, further improvement of the eczema and absence of pustules were reported after 1 month. At visit 3, 2 months later, an increase of the eczema was reported. This was probably related to a lack of adherence since our patient used the corticosteroid cream, tacrolimus ointment, and emollient inconsistently. The legs showed dry eczematous lesions and 
postinflammatory hyperpigmentation. However, pustules were still absent. Cultures of the skin taken at visits 1,2 , and 3 were all positive for $S$. aureus.

\section{Discussion}

This case series describes the successful treatment of $S$. aureus-related dermatoses with an antibacterial endolysin, which is currently available as an over-the-counter medical device. All 3 patients had recurrent $S$. aureus infections despite adequate antibiotic treatment. Atopic predisposition and nasal carriage of $S$. aureus probably contributed to the chronicity of their symptoms $[2,3,11]$. Despite the improvement of symptoms in these patients, $S$. aureus was still cultured from their skin during and after treatment with Staphefekt. We believe that Staphefekt induces a clinically relevant reduction of $S$. aureus rather than a total eradication. In addition, it is well-known that the skin lesions can get recolonized quickly due to constant exposure to $S$. aureus from the nose and environment. Apparently, complete and sustained eradication is not necessary to achieve clinically relevant improvement with an endolysin-based topical treatment. Since the results from our cultures are only qualitative in nature, future studies should further investigate the effect of Staphefekt on the S. aureus load and composition of the microbiome, using quantitative methods and sequencing technologies.

Clinical symptoms quickly reappeared both after stopping antibiotics (cases 1 and 2) and Staphefekt (case 1), likely due to rapid $S$. aureus recolonization to a clinically relevant state. The positive clinical effect after restarting $S$. aureus suppression with Staphefekt in case 1 supports this hypothesis. Concurrent treatment of the nostrils combined with treatment of the complete skin surface could potentially further reduce recolonization.

It should be noted that both impetiginized eczema and folliculitis have a naturally fluctuating disease course, which complicates the interpretation of our data. However, all 3 patients visited our outpatient clinic frequently with recurring and continuous symptoms (despite adequate treatment) for long periods already before the start of Staphefekt treatment $(10,3.5$, and 13 months). This suggests that remission is likely due to the use of Staphefekt rather than to chance.

Although limited by its uncontrolled design, this case series illustrates that topical therapy with Staphefekt SA.100 to continuously suppress $S$. aureus can lead to the reduction and prevention of clinical symptoms in 3 cases of $S$. aureus-related skin conditions, folliculitis and impetiginized dermatitis. Importantly, Staphefekt targets both methicillin-sensitive and methicillin-resistant $S$. aureus but does not interfere with the commensal skin microbes and is also not expected to induce bacterial resistance due to its specific mechanism of actions [8, 9]. It could therefore be considered as a feasible alternative for S. aureus-related superficial skin infections, since classical (long-term) antibiotic therapy is clearly associated with the induction of bacterial resistance and other adverse effects $[4,5]$. The observed beneficial clinical effect of Staphefekt in these cases is encouraging, and further study of its efficacy and safety is warranted in randomized controlled clinical trials. 
Totté et al.: Successful Treatment of Chronic Staphylococcus aureus-Related Dermatoses with the Topical Endolysin Staphefekt SA.100: A Report of 3 Cases

\section{Statement of Ethics}

The patients described in this case report provided informed consent.

\section{Disclosure Statement}

The Department of Dermatology of the Erasmus MC University Medical Center Rotterdam received an unrestricted grant from Micreos Human Health, the Netherlands, to perform clinical studies with Staphefekt SA.100. Micreos Human Health provided Staphefekt SA.100 for the patients.

\section{References}

1 McCaig LF, McDonald LC, Mandal S, Jernigan DB: Staphylococcus aureus-associated skin and soft tissue infections in ambulatory care. Emerg Infect Dis 2006;12:1715-1723.

2 Laureano AC, Schwartz RA, Cohen PJ: Facial bacterial infections: folliculitis. Clin Dermatol 2014;32:711-714.

3 Brüssow H: Turning the inside out: the microbiology of atopic dermatitis. Environ Microbiol 2016;18:2089-2102.

-4 van Bijnen EM, Paget WJ, den Heijer CD, Stobberingh EE, Bruggeman CA, Schellevis FG, et al: Primary care treatment guidelines for skin infections in Europe: congruence with antimicrobial resistance found in commensal Staphylococcus aureus in the community. BMC Fam Pract 2014;15:175.

5 World Health Organization: Antimicrobial resistance. Global report on surveillance: World Health Organization (WHO). 2014. http://www.who.int/drugresistance/documents/surveillancereport/en/. Hepburn L, Hijnen DJ, Sellman BR, Mustelin T, Sleeman MA, May RD, et al: The complex biology and contribution of Staphylococcus aureus in atopic dermatitis, current and future therapies. Br J Dermatol 2016, Epub ahead of print.

7 Pastagia M, Schuch R, Fischetti VA, Huang DB: Lysins: the arrival of pathogen-directed anti-infectives. J Med Microbiol 2013;62:1506-1516.

8 Herpers BL, Badoux P, Pietersma F, Eichenseher F, Loessner MJ: Specific lysis of methicillin susceptible and resistant Staphylococcus aureus by the endolysin Staphefekt SA.100 TM. Abstract R144. 24th European Congress of Clinical Microbiology and Infectious Diseases (ECCMID), Barcelona, Spain, 2014.

9 Herpers BL, Badoux P, Totté JEE, Pietersma F, Eichenseher F, Loessner MJ: Specific lysis of Staphylococcus aureus by the bacteriophage endolysin Staphefekt SA.100: in vitro studies and human case series. In: Abstract book of the EuroSciCon meeting antibiotic alternatives for the new millennium, November 5-7, 2014, London, UK, pp 8-9 (cited May 17, 2017). Available from: http://lifescienceevents.com/wp-content/uploads/AntibioticAlternatives57thNovABSTRACTS2014.pdf.

10 Totté JEE, van den Boogaard M, Pardo Cortes LM, Pasmans SGMA: Staphylococcus aureus specific topical treatment of rosacea and acne with the endolysin Staphefekt SA.100. Abstract P1212. 24th European Academy of Dermatology and Venereology Congress, Copenhagen, Denmark, 2015.

11 Wertheim HF, Melles DC, Vos MC, van Leeuwen W, van Belkum A, Verbrugh HA, et al: The role of nasal carriage in Staphylococcus aureus infections. Lancet Infect Dis 2005;5:751-762.

12 ClinicalTrials.gov. The effect of Gladskin on disease severity and the skin microbiome, including Staphylococcus aureus, in patients with atopic dermatitis. Identifier NCT02840955. Bethesda, National Library of Medicine, July 11, 2016 (cited May 17, 2017). Available from: https://www.clinicaltrials.gov/show/NCT02840955. 


\section{Case Reports in Dermatology}
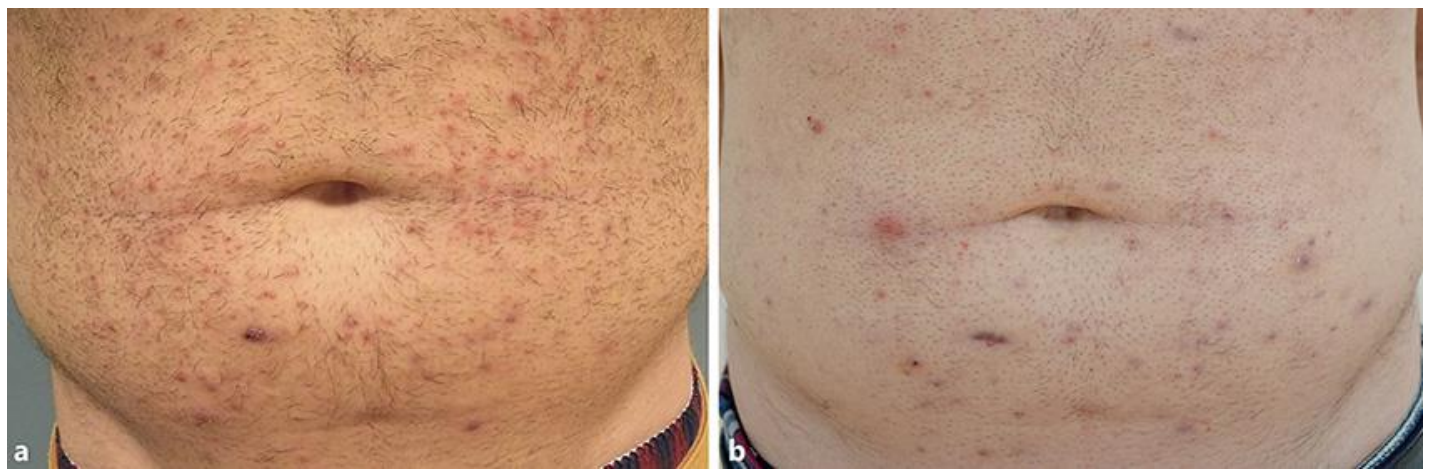

Fig. 1. Folliculitis. a Baseline; extensive follicular papules and pustules. b After 8 weeks; few follicular papules, postinflammatory hyperpigmentation, and scarring.
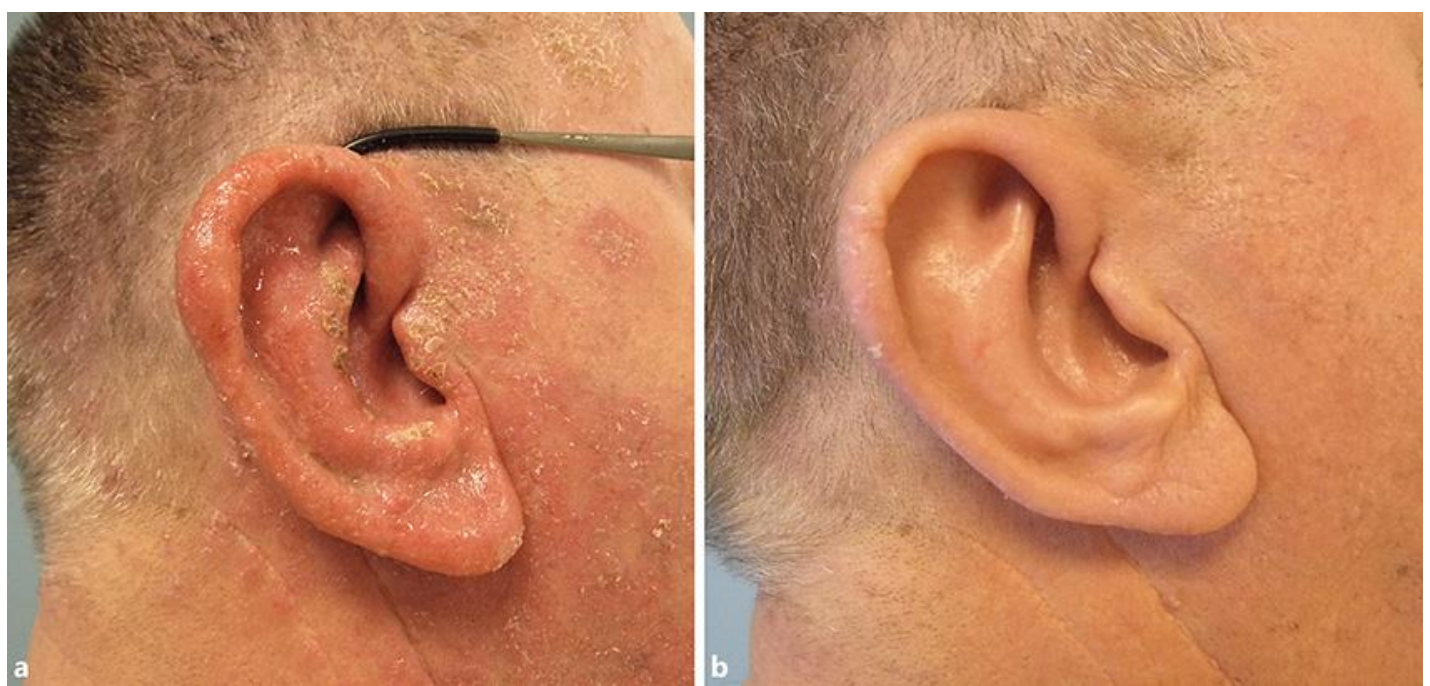

Fig. 2. Nummular eczema with secondary infection. a Baseline; erythematosquamous nummular lesions forming confluent plaques covered with crustae and swelling of the ear. b Six weeks later; very mild erythematous macula with minimal scaling. 
Totté et al.: Successful Treatment of Chronic Staphylococcus aureus-Related Dermatoses with the Topical Endolysin Staphefekt SA.100: A Report of 3 Cases

Table 1. Overview of in vitro and clinical studies with Staphefekt

\begin{tabular}{|c|c|c|}
\hline Authors, year & Published in & Summary of findings \\
\hline $\begin{array}{l}\text { Herpers et al., } \\
2014 \text { [8] }\end{array}$ & $\begin{array}{l}\text { Abstract R144 at the 24th } \\
\text { European Congress of Clinical } \\
\text { Microbiology and Infectious } \\
\text { Diseases (ECCMID), Barcelona }\end{array}$ & $\begin{array}{l}\text { Lysis of } S \text {. aureus by Staphefekt is dose dependent, specific, and efficient, } \\
\text { compared to control strains of coagulase-negative staphylococci (in vitro) } \\
\text { MSSA and MRSA proved to be equally susceptible to the endolysin } \\
\text { Induction of resistance against Staphefekt could not be achieved in vitro }\end{array}$ \\
\hline $\begin{array}{l}\text { Herpers et al., } \\
2014 \text { [9] }\end{array}$ & $\begin{array}{l}\text { Summary of presentation at } \\
\text { EuroSciCon meeting, London }\end{array}$ & $\begin{array}{l}\text { In } 3 \text { out of } 7 \text { patients with rosacea, } S \text {. aureus was cultured from a lesion; } \\
\text { after the local application of Staphefekt for } 1 \text { week, S. aureus was } \\
\text { eradicated from the lesion in all } 3 \text { patients } \\
\text { In } 6 \text { out of } 8 \text { patients with recurring dermatitis ( } 3 \text { constitutional eczema, } \\
2 \text { chronic contact dermatitis, and } 1 \text { perioral dermatitis), S. aureus was } \\
\text { found in skin cultures before treatment; in } 5 \text { of } 6 \text { patients, symptoms } \\
\text { decreased during treatment with Staphefekt, and patients reported less } \\
\text { need of corticosteroids }\end{array}$ \\
\hline $\begin{array}{l}\text { Totté et al., } \\
2015 \text { [10] }\end{array}$ & $\begin{array}{l}\text { Abstract P1212 at } 24 \text { th EADV } \\
\text { Congress, Copenhagen }\end{array}$ & $\begin{array}{l}\text { Patients with rosacea }(n=112) \text { and acne }(n=56) \text { reported an } \\
\text { improvement of inflammation-related symptoms and quality of life } \\
\text { (Skindex-29) after } 6 \text { weeks of topical treatment with Staphefekt SA.100 in } \\
\text { a prospective questionnaire study }\end{array}$ \\
\hline $\begin{array}{l}\text { Pasmans et al., } \\
2016 \text { [12] }\end{array}$ & $\begin{array}{l}\text { Study protocol randomized } \\
\text { controlled trial, registered at } \\
\text { ClinicalTrials.gov, } \\
\text { NCT02840955 }\end{array}$ & $\begin{array}{l}\text { Ongoing double-blinded randomized controlled trial aiming to assess the } \\
\text { difference in the need for corticosteroid cotherapy between the } \\
\text { Staphefekt and a placebo group over a } 12 \text {-week treatment period } \\
\text { Secondary objectives include differences in clinical efficacy, quality of life, } \\
\text { microbial composition, and safety and tolerability }\end{array}$ \\
\hline
\end{tabular}

